\title{
Differentiating 3D textile composites: A novel field of application for Digital Volume Correlation
}

\author{
Arturo Mendoza ${ }^{\mathrm{a}, \mathrm{b}, *}$, Julien Schneider ${ }^{\mathrm{c}}$, Estelle Parra ${ }^{\mathrm{b}}$, Enrico Obert $^{\mathrm{b}}$, \\ Stéphane Roux ${ }^{\mathrm{a}}$ \\ ${ }^{a} L M T$ (ENS Paris-Saclay/CNRS/Univ. Paris-Saclay), \\ 61 Avenue du Président Wilson, 94235 Cachan, France \\ ${ }^{b}$ Safran Tech, Rue des Jeunes Bois, 78772 Magny les Hameaux, France \\ ${ }^{c}$ Safran Aircraft Engines, Rond Point Réné Ravaud - Réau, 77550 Moissy-Cramayel, France
}

\begin{abstract}
Digital Volume Correlation is an appealing technique for establishing a novel comparison (differentiating) tool based on full field measurements of structured materials such as $3 \mathrm{D}$ woven composites. This approach provides a quantifiable description of the weaving distortions on woven composites. It also offers applications ranging from the quantification of the influence of different weaving conditions, up to nondestructive testing of composite parts. The method is validated on real 3D woven composite samples revealing both "metric differences" (deformations of a textile with respect to reference one) and "topological differences" (such as the occurrence of missing yarns).
\end{abstract}

Keywords: Woven Composites, Textiles, Digital Volume Correlation, Tomography

\section{Introduction}

Woven composites are fast becoming key in many industries such as aeronautical and aerospace mainly due to their very attractive specific properties (e.g. strength to weight ratio). Particularly, 3D woven composites (three-dimensional weaving pattern) have appeared in many new applications requiring high mechanical properties such as the turbomachine fan blade and casing developed for the LEAP motor [1]. Evidently the increasing interest in these materials has generated a high demand for proper characterization methods, accurate FE simulations, suitable nondestructive testing (NDT) techniques, and adequate visualization techniques.

\footnotetext{
${ }^{*}$ Corresponding author

Email address: arturo.mendoza.quispe@lmt.ens-cachan.fr (Arturo Mendoza)
} 
The structured nature of woven composites, as described by their weaving pattern, allows for a new type of analysis: namely one that takes into account their topology. This powerful statement exploits the property that different woven samples (sharing a weaving pattern) can be related by means of continuous displacements.

The quantitative analysis of such deformations is of great interest for woven composites. For instance, the evaluation of yarn displacements and deformations, such as stretching and bending, may help in defining acceptable or unacceptable weaving distortions. Furthermore, this analysis could help identify the step in the manufacturing process or the set of parameters in need of optimization so as to avoid undesired results [2]. Here, the evaluation is carried out at the meso-scale, which implies that any phenomena occurring at the micro-scale is neglected [3].

This quantitative characterization drastically improves over qualitative methods that do not provide enough information to detect subtle deformations and even not so subtle ones. Thereby, image correlation can be used as a compelling NDT technique for the manufacturing of high technology composite materials.

Here is presented the effectiveness of image correlation for registering pairs of tomographic volumes of $3 \mathrm{D}$ woven composites. This allows the measurement of "metric differences" as well as the so-called "topological differences". The former quantify all yarn displacements and deformations in the studied sample. And the latter encompass all weaving anomalies that cannot be eliminated by a simple continuous deformation of the medium, such as loops and missing (or extra) yarns.

The paper is structured as follows. Section 2 presents the mathematical framework of the image correlation technique. Section 3 recalls briefly the adaptation of Digital Volume Correlation (DVC) for the analysis of woven composites, as well as some additional analysis tools. The robustness of the approach is demonstrated in Section 4 with two challenging examples that target the "metric differences" and "topological differences" respectively. Finally, some conclusions and outlook are given in Section 5.

\section{Image correlation}

Digital Image Correlation (DIC) [4] is a popular technique to accurately measure 2D displacements fields from images pairs. As an extension of standard DIC, Digital Volume Correlation (DVC) [5] is a fast growing technique [6] that measures the internal displacement fields from a pair of volumes (3D images). As a true three dimensional technique, DVC is distinct from the 3D surface deformation methods that rely on two-angle planar images (stereo-correlation [7]).

The analysis of displacement fields from mechanical tests is a key ingredient to bridge the gap between experiments and simulations. It also allows the

subsequent extraction of mechanical properties or quantitative evaluations of constitutive law parameters $[8,9]$, one of the most important steps of the analysis 
in Solid Mechanics [10]. This explains the increasing interest of measuring the displacement fields of surfaces or volumes belonging to stressed specimens and structures using images acquired at different stages of loading. As such the displacement field is measured by means of the observed image texture that is assumed to passively follow the displacement of the analyzed solid. It is desirable that this texture have strong contrasts so as to be sensitive to small displacements. DVC usually relies on the natural texture of the studied material as a basis for correlation [11] since most procedures used to improve the contrast of the material texture will also modify its mechanical properties (which may be of interest).

DIC and DVC approaches can be of local [5] or global $[12,13]$ nature. The former evaluates the apparent mechanical transformation through a large number of independent analyses on sub-images or correlation windows, while the latter does so over the entire region of interest (ROI). In reference [14], it is shown that global approaches generally out-perform the local ones thanks to the embedded continuity requirements which help capturing more complex displacements fields.

\subsection{Correlation procedure}

The registration between the reference configuration $f(\boldsymbol{x})$ and the deformed configuration $g(\boldsymbol{x})$ is based on the brightness conservation assumption [13]

$$
f(\boldsymbol{x}) \approx \tilde{g}(\boldsymbol{x})
$$

with

$$
\tilde{g}(\boldsymbol{x}) \equiv g(\boldsymbol{x}+\boldsymbol{u}(\boldsymbol{x}))
$$

where $\boldsymbol{u}$ is the Lagrangian displacement vector field applied at any material point $\boldsymbol{x}$, and $\tilde{g}(\boldsymbol{x})$ defines the corrected (back-deformed) configuration,

The goal is to find the displacement $\boldsymbol{u}$ needed to reach full coincidence of $f$ and $\tilde{g}$ by minimizing the squared norm of residuals $\eta$, defined as

$$
\eta=f-\tilde{g}
$$

This is an ill-posed problem since the available information (intensity levels) is insufficient to uniquely determine a vector displacement field. For such a reason, $\boldsymbol{u}$ is restricted to a space of low dimensionality, which results in a wellposedness and (depending on the choice of this space) a good conditioning of the problem. A convenient choice is the decomposition of $\boldsymbol{u}$ on a set of well chosen kinematics fields $\psi_{i}$ (forming a basis), such as those used in the framework of the finite element (FE) method

$$
\boldsymbol{u}(\boldsymbol{x}) \approx \sum a_{i} \psi_{i}(\boldsymbol{x})
$$

Then, the overall solution is given by the vector $\{\boldsymbol{a}\}$ containing the amplitudes that minimize the squared norm of the residuals $\eta$ over the region of interest $\Omega$.

$$
\tau=\int_{\Omega} \eta^{2} \mathrm{~d} \boldsymbol{x}
$$


The superposition of the chosen kinematics basis over $\Omega$ makes the sought amplitudes $a_{i}$ interdependent, hence the term "global".

Finally, a classical Newton-Raphson routine [15], an iterative linearised optimization procedure, is used to solve for

$$
\frac{\partial \tau}{\partial\{\boldsymbol{a}\}}=0
$$

which leads to the linear system:

$$
[\boldsymbol{M}]\{\delta \boldsymbol{a}\}=\{\boldsymbol{b}\}
$$

with

$$
\boldsymbol{M}_{i j}=\int_{\Omega}\left(\boldsymbol{\nabla} f \cdot \psi_{i}\right) \cdot\left(\nabla f \cdot \psi_{j}\right) \mathrm{d} \boldsymbol{x}
$$

and

$$
\boldsymbol{b}_{i}=\int_{\Omega}\left(\boldsymbol{\nabla} f \cdot \psi_{i}\right) \cdot(f-\tilde{g}) \mathrm{d} \boldsymbol{x}
$$

As such, the unknown degrees of freedom are updated with:

$$
\{\boldsymbol{a}\}^{k+1}=\{\boldsymbol{a}\}^{k}+\{\delta \boldsymbol{a}\}
$$

This allows to recompute $\tilde{g}$ and $\eta$ until convergence is reached.

\subsection{Relaxed brightness conservation}

Sometimes, the brightness conservation assumption is not respected due to factors extrinsic to the displacement. Typically, such changes account for light source intensity variations or spurious reflections induced by the geometry. Additionally, these changes can also have some physical meaning such as oxidation or wetting. In the case of volumetric analysis, these changes can originate from the acquisition method. In particular for tomography, effects such as the ring artefact or the cupping artefact (resulting from the phenomenon known as "beam hardening" in which outer elements seem to be more absorbing) need to be taken into account.

The relaxed brightness conservation assumption redefines the corrected image as:

$$
\tilde{g}(\boldsymbol{x}) \equiv g(\boldsymbol{x}+\boldsymbol{u}(\boldsymbol{x}))+v(\boldsymbol{x}, g(\boldsymbol{x}+\boldsymbol{u}(\boldsymbol{x})))
$$

where

$$
v(\boldsymbol{x}, h) \approx \sum_{p} v_{p}(\boldsymbol{x}) \cdot h^{p}(\boldsymbol{x})
$$

For simplicity, the functional $v(\boldsymbol{x}, \cdot)$ is applied over the reference image $f$ and not over the test image $\tilde{g}$. This implies handling a constant Hessian matrix (equivalent to being computed only at convergence where $\tilde{g}$ approximates $f$ ), which accelerates the optimization procedure. Additionally, it is usual to limit the correction up to the second order. This is written as:

$$
\tilde{g}(\boldsymbol{x}) \equiv g(\boldsymbol{x}+\boldsymbol{u}(\boldsymbol{x}))+v_{0}(\boldsymbol{x})+v_{1}(\boldsymbol{x}) \cdot f(\boldsymbol{x})
$$


Traditionally, the first and second order corrections $\left(v_{0}(\boldsymbol{x})\right.$ and $\left.v_{1}(\boldsymbol{x})\right)$ are called "brightness" and "contrast" respectively, since such are the phenomena they describe in the surface of a sample. However, such terminology is not suited for the volumetric case since $f$ refers to the absorption of X-rays. Nonetheless, due to their standard use in the field of image analysis, they will be kept in the following as such.

Following the same development as previously shown, equations (8) and (9) become:

$$
\boldsymbol{M}_{i j}=\int_{\Omega}\left(\Phi_{i}(f) \cdot \psi_{i}\right) \cdot\left(\Phi_{i}(f) \cdot \psi_{j}\right) \mathrm{d} \boldsymbol{x}
$$

and

$$
\boldsymbol{b}_{i}=\int_{\Omega}\left(\Phi_{i}(f) \cdot \psi_{i}\right) \cdot(f-\tilde{g}) \mathrm{d} \boldsymbol{x}
$$

where the type of degree of freedom associated to $i$ defines

$$
\Phi_{i}(f)= \begin{cases}\nabla f, & \text { if associated to displacement } \\ 1, & \text { if associated to brightness } \\ f, & \text { if associated to contrast }\end{cases}
$$

This allows to identify the type of correction associated to each degree of freedom enumerated by index $i$. Then the resolution of the problem follows the aforementioned iterative scheme.

Finally, it should be noted that while the correlation procedure does solve for the correction fields (i.e. $\boldsymbol{u}(\boldsymbol{x}), v_{0}(\boldsymbol{x})$ and $v_{1}(\boldsymbol{x})$ ), the correction proposed by only the displacement can be computed at any time. Hence, the following notation is proposed in order to accommodate equations (2) and (13)

$$
\tilde{g}_{u}(\boldsymbol{x}) \equiv g(\boldsymbol{x}+\boldsymbol{u}(\boldsymbol{x}))
$$

and

$$
\tilde{g}_{u v}(\boldsymbol{x}) \equiv \tilde{g}_{u}(\boldsymbol{x})+v_{0}(\boldsymbol{x})+v_{1}(\boldsymbol{x}) \cdot f(\boldsymbol{x})
$$

with the correlation residuals based on kinematics only

$$
\eta_{u}=\tilde{g}_{u}-f
$$

and based on the full correction

$$
\eta_{u v}=\tilde{g}_{u v}-f
$$

with the optimization being performed on the squared norm of $\eta_{u v}$.

\subsection{Mechanical regularization}

Regularization techniques can be used in order to further condition the DVC problem, as well as to reduce the measurement uncertainty. While a classical soft Tikhonov regularization [16] could be used, it is preferred to introduce a penalization based on the "distance" between the estimated displacement field 
and that of the solution to a homogeneous elastic problem [17, 18]. This distance is known as the equilibrium gap [19] which assumes an elastic behavior at a small scale and a boundary regularization [13, 20, 21].

Then, by defining the strain tensor $\varepsilon$ as the measure of deformation inside the material

$$
\varepsilon=\frac{1}{2}\left(\nabla \boldsymbol{u}+\nabla \boldsymbol{u}^{\top}\right)
$$

the relevant state of an elastic medium can be described by the constitutive equation

$$
\boldsymbol{\sigma}=\boldsymbol{C}: \varepsilon
$$

where the (Cauchy) stress tensor $\boldsymbol{\sigma}$ is related to the strain tensor $\boldsymbol{\varepsilon}$ by means of the elasticity tensor $\boldsymbol{C}$ (also called Hooke tensor), which summarizes the constitutive law of the elastic material.

As such, the displacement field $\boldsymbol{u}(\boldsymbol{x})$ in the bulk of an elastic body can be described with the Lamé operator:

$$
\operatorname{Lam}(\boldsymbol{u}) \equiv \nabla \cdot \boldsymbol{\sigma}
$$

which, in the context of the FE method can be expressed using the stiffness matrix $\boldsymbol{K}$ as nodal forces

$$
[\boldsymbol{K}]\{\boldsymbol{u}\}=\left\{\boldsymbol{F}_{\boldsymbol{n}}\right\}
$$

and in the absence of body forces $\boldsymbol{F}_{\boldsymbol{n}}$, the equilibrium equations read:

$$
[\boldsymbol{K}]\{\boldsymbol{u}\}=0
$$

Then, the minimization of the quadratic norm of $\operatorname{Lam}(\boldsymbol{u})$, the so-called "equilibrium gap", effectively penalizes any deviation and mechanically regularizes the sought displacement field $\boldsymbol{u}$. The standard functional defined in equation (5) is supplemented with this new functional. Nonetheless, the nature of the (second order differential) Lamé operator calls for a proper weighting scheme. Such is obtained with a factor proportional to the fourth power of a regularization length, $\xi^{4}$ as shown by dimensional analysis.

It is important to note that this strategy does not require the studied volume to strictly obey linear elasticity. Rather it can be seen as a filter that locally dampens abrupt displacement gradients (high spatial frequencies) in order to guarantee a smooth and differentiable displacement field. Such filter is tuned by means of the regularization length $\xi$, which, if smaller than the finite element size, nullifies the mechanical regularization. In this case, the benefit of referring to elasticity is a natural way to ensure Galilean invariance.

Furthermore, this technique can be adapted for the regularization of the gray level correction fields presented previously. For such, it suffices to substitute the Lamé operator with the Laplace operator

$$
\Delta v \equiv \nabla^{2} \cdot v
$$

As such, two new functionals are derived from the quadratic norm of $\boldsymbol{\Delta} v_{0}$ and $\boldsymbol{\Delta} v_{1}$. The optimization procedure follows identically to the previous. Finally, 
two regularization lengths are employed: $\xi_{u}$ for the displacement field and $\xi_{v}$ for the gray level correction fields $\left(\xi_{v_{0}}\right.$ and $\xi_{v_{1}}$ are chosen to be equal).

It should be noted that $\xi_{u}$ and $\xi_{v}$ need to be tuned so as to achieve a good balance between a well-posed problem not prone to noise and a problem with enough flexibility (freedom). In fact, too short $\xi_{u}$ and $\xi_{v}$ may lead to a confusion between the corrections related to displacement and those related to gray level modifications. The choice of these parameters should be guided by a correct interpretation of the results as well as of the obtained residuals.

\subsection{Multiscale analysis}

Since it is possible to compute the cost function $\tau$ and its gradients for any set of parameters $\boldsymbol{a}$, the optimization can (in principle) be performed with any iterative numerical algorithm which should more or less quickly converge to a solution. In most cases, the convergence towards the local minimum closer to the initial guess is of concern. Many different strategies have been employed in that sense [22]. Particularly, a multiscale algorithm [23] has been shown to be extremely robust and capable to benefit from an optimization by descent while limiting the effects of spurious local minima. This method has shown good results for local and global approaches [10].

The idea behind the multiscale algorithm is to start with strongly low-pass filtered (smoothed) images in order to artificially enlarge the effective correlation length and hence the basin of attraction of approximate displacement determinations. The algorithm is repeatedly applied over less filtered images (gradually restoring the details), using the displacement obtained from the coarser images as an initial guess. The recursion stops when the original image is recovered.

\subsection{Image of residuals}

The image of residuals $\eta$ is a useful criterion to evaluate (qualitatively or quantitatively) the obtained solution. Obviously, if the registration were perfect, the residuals would contain nothing but image noise. Usually they are found to be a small fraction of the full dynamic range of the gray levels. Moreover, the residual field allows correlation errors to be spatially located. They also help to quickly verify the proper convergence of the algorithm, and to possibly correct or extend the chosen kinematic basis.

\subsection{Discretisation effects}

It should be noted that while $f$ and $g$ are the discretisation of a continuous phenomenon, they have been treated as continuous functions by means of interpolation schemes; this is implicitly expressed in equation (1). Such formulation requires the intensity values in the deformed image at arbitrary points $\boldsymbol{x}+\boldsymbol{u}(\boldsymbol{x})$. The errors induced by this interpolation take the form of an over- or under-estimation of the displacement components. The average of these errors exhibits a symmetry with respect to the half-pixel displacement [24], which can be used to determine the a priori level of errors and uncertainty to be expected from of the interpolation scheme. It may be worth adding that because of the 
expected differences at very small scales, it makes no sense to spend time for a very accurate interpolation. Hence, the trilinear interpolation is suitable.

Finally, the choice of a discretisation method for the displacement field is crucial as a good spatial resolution will be accompanied by a high displacement uncertainty and viceversa. Finer meshes induce a better spatial resolution but displacement uncertainties are larger $[10,25]$ because of the fewer pixels per kinematic degree of freedom considered in the analysis.

\section{Multiresolution isotropic approach}

The hierarchy of information is an important notion in image analysis. This happens due to a natural nesting of several objects or features (of different sizes) within a single image. Developing robust methods capable of dealing with fine details - which usually are not a highly discriminant source of informationwhile also coping with broad definitions and still remain numerically efficient, proves to be a complex task. Coarse-to-fine resolution strategies often provide a simple yet effective solution. These include the traditional (Gaussian) pyramidal approach [26] or the more recent scale space theory [27], both being strongly related. The pyramidal method uses the results obtained on a lower resolution image to guide the processing on a higher resolution image, repeatedly until the original image is reached. Thus, the coarser levels will help to capture the largest displacements, which will be iteratively refined at each finer resolution level.

Additionally, this multiresolution strategy aligns perfectly with the multiscale analysis presented in section 2.4. While the former deals with the different scales of interest present in the textile, the latter ensures algorithmic speed up and convergence.

Moreover, when dealing with displacements, the notion of anisotropy should be addressed in the image analysis. For the present case study, the yarn crosssections are of great interest. Though initially (in the manufacturing process) approximately circular in shape, the yarn cross-sections on the final textile are greatly flattened, thus giving rise to elongated structures. In fact, they are commonly modelled after ellipses or lenticular shapes [28]. As such, similar displacements in different directions will not have the same impact. A straightforward solution is a simple geometric transformation in order to make the image isotropic, where yarn cross-sections approximate a circular shape. However, this affinity does not need to be exceptionally "precise", but rather approximate since the goal is to obtain in average more isotropic shapes. A similar argument can be made for textiles with more complex shapes. While in such case the anisotropy removal may be less effective, it remains beneficial for the aforementioned reasons.

Finally, the multiresolution isotropic approach is pursued by first removing the anisotropy using a convenient scaling factor (per axis), followed by the construction of a Gaussian pyramid from the now isotropic image. Here the pyramid levels are constructed up to the point where the smallest scale remains distinguishable. In this case, the smallest case is the yarn cross-section. An 
example of the procedure can be seen in figure 1. Additionally, the calculations are only performed on the isotropic levels of the pyramid. The obtained results can be extrapolated to the anisotropic case if needed.

Subsequently, the correlation procedure is applied on each pyramid level and with the lower levels providing the initialization for the upper levels. The DVC technique used here is based on the relaxed brightness conservation assumption, with a displacement field mechanically regularized (using the previously described elastic behavior), and a gray level correction field regularized by penalizing the Laplacian obtained on each scalar field independently.

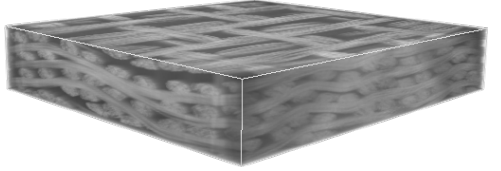

(a)

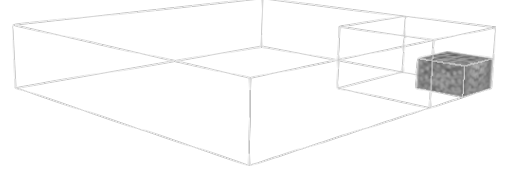

(c)

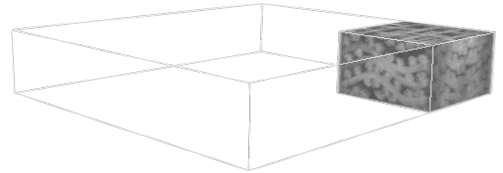

(b)

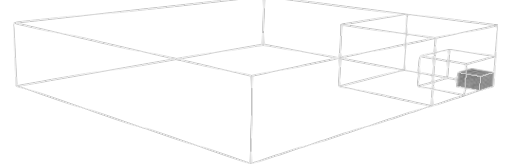

(d)

Figure 1: Volumes constructed for the multiresolution isotropic approach. The original (anisotropic) volume is shown in (a) while the first three levels of the (isotropic) pyramid are shown from (b) to (d).

\subsection{Metric differences}

The found displacement field allows a quantitative analysis through the measurement of the existing "metric differences" between the studied samples. Such differences highlight the relative yarn displacements needed to accommodate the test sample towards the reference one.

Furthermore, the displacement field can be used to compute a powerful descriptor: the relative strain. It describes the deformation in terms of relative displacement and excludes rigid-body motion of the medium. This descriptor, while numerically identical to the strain definition given in continuum mechanics, proposes a novel key concept: it does not relate different configurations for a single medium, but rather different configurations corresponding to different media. Thus the relative nature of the descriptor.

The convention used here for obtaining the relative strain is that of the logarithmic strain, also known as true strain or Hencky strain [29]. This definition accounts for large strains as well as large for large rotations [30]. As such, the obtained symmetric tensor $\varepsilon$ describes normal and shear phenomena in a given coordinate system (e.g. Cartesian). Additional useful tools (for the small strain assumption) are the tensor invariants which remain constant despite of the reference frame. These can be used to obtain the positive invariant quantity known 
as the equivalent [31, 32] or Von Mises strain

$$
\varepsilon_{e q}=\frac{2}{3} \sqrt{I_{1}^{2}-3 I_{2}}
$$

where

$$
I_{1}=\operatorname{tr}(\varepsilon)
$$

and

$$
I_{2}=\frac{1}{2}\left\{\left[\operatorname{tr}(\varepsilon)^{2}\right]-\operatorname{tr}\left(\varepsilon^{2}\right)\right\}
$$

are respectively the first and second invariants of the strain tensor $\varepsilon$. These useful invariant indicators account respectively for volume changes through $\operatorname{tr}(\varepsilon)$, as well as for shear at constant volume through $\varepsilon_{e q}$.

Finally, the set of indicators presented here will be used in the following because of their ability to describe the targeted applications. However, the means in which the "metric differences" can be exploited are not limited to just these indicators. In fact, it is imperative that they be regarded with respect to the application or context in mind. Awareness on this subject will reinforce the importance of "metric differences" for composite materials. For instance, composite manufacturing can benefit considerably from proper quantitative indicators by allowing a precise feedback on yarn displacements and deformations [33] or by doting them with some predictive capabilities over the final behavior on the manufactured sample.

\subsection{Topological differences}

As it was previously introduced, woven composite materials share a key intrinsic information: the underlying weaving pattern. Such weaving pattern is represented in a unit cell, which is then tessellated (tiled) to form a full scale textile. Hence, this procedure of correlation on woven composites is based on the assumption of a constant topology: properties preserved through deformations, twisting, and stretching (tearing and closing are not allowed).

Hence, in principle, different specimens made with the same weaving pattern can always be set into correspondence using a continuous displacement field. And whenever this assumption is invalidated, the so-called "topological differences" come into sight. Such event will occur in the presence of weaving anomalies (loops, missing yarns) between the studied volumes since no continuous displacement field is capable of bringing the volumes into full coincidence.

It is important to note that the procedure (and the assumption of topology) is only valid between regions of interest $\Omega$ (of arbitrary size and shape) containing the same sample of the weaving pattern, i.e. containing the same textile, as shown in figure 2 .

Finally, the "topological differences" are evaluated through the image of residuals which accounts only for the found displacement field, as in equation (19). The proper analysis of the residuals will showcase high-valued regions which are not dispersed at random, but rather highly structured and meaningful. Moreover, multiple indicators can be envisaged from these "topological differences". 


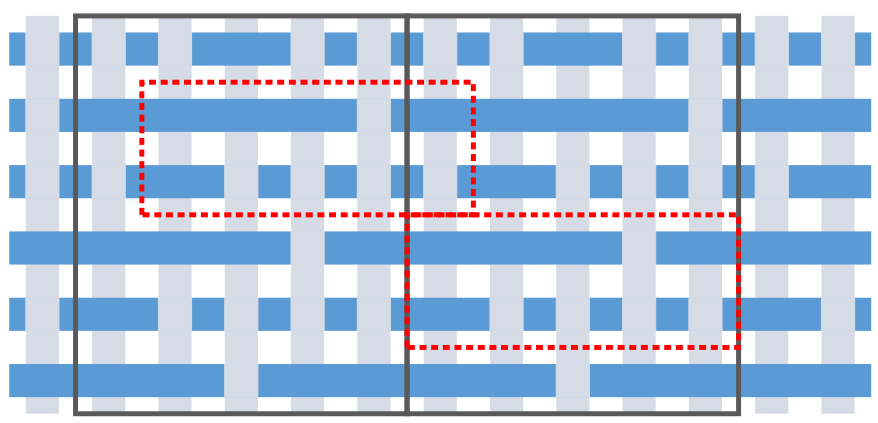

Figure 2: Sample textile showing multiple tessellated '-' the arrangement of warp and weft yarns, which define some topologically equivalent $\square$ regions of interest $\Omega$.

These can in turn be fed as low level features into specialized image processing or machine learning algorithms for the segmentation, classification and identification of structures of interest. Possible applications of such indicators fall within the realm of NDT tools used on composite materials.

\section{Results}

Two pairs of 3D woven composites specimens are used in the present analysis. These samples were obtained through X-ray micro-tomography and under similar acquisition conditions. Since the analysis is performed at the meso-scale, the chosen image resolution is coarse enough so as to "average" the information at the micro-scale (i.e., the carbon fibers), while being sufficiently fine so as to allow a proper description of the yarn itself. Furthermore, the acquisition is performed with low energy X-rays in order to get the maximum differentiation between the (carbon) yarns and the (polymer) resin. This helps obtaining (after reconstruction) very different gray levels for each phase, despite the fact that both have very close atomic numbers. Finally, all imaged volumes contain an entire unit cell and the reference configuration for each studied pair is chosen at random.

The first pair of volumes corresponds to two preform specimens manufactured after a given weaving pattern. These specimens only contain the woven yarns, i.e. the reinforcement phase of the composite. Neither of the specimens exhibit any anomaly. On the other hand, being different samples, their yarn disposition is not strictly identical. Thus, measuring their "metric differences" and extracting useful indicators such as the relative strainsis of great interest.

The second pair of volumes corresponds to two injected specimens manufactured after a given weaving pattern different from the first pair. These specimens contain some intentional weaving anomalies. One of the specimens was manufactured with two missing yarns, while the other was manufactured with 
one missing yarn (different from the previous two), clearly incurring into a disagreement with the assumption of topology. Hence measuring their "topological differences" and analyzing their residuals will be a key point.

Both pairs of volumes undergo the multiresolution isotropic approach with similar parameters. First of all, appropriate anisotropic scale factors are chosen for each volume pair. Then, the image pyramids are constructed with as many levels as needed: 5 levels for the first pair and 4 for the second. For all levels in a pyramid, the kinematic basis is described using a single structured regular mesh composed of only cube-shaped finite elements (H8). In particular, the element size at the smallest scale is set to $2 \times 2 \times 2$ voxels and scales accordingly with the pyramid levels. Then the meshes are constructed so as to fill up the entire workable area. Furthermore, the regularization lengths are defined at the highest scale and kept constant for all levels. Since they do not scale, the correlation perceives an effect of regularization relaxation progressively from lower to higher levels. The regularization lengths concerned with the displacement field $\xi_{u}$ are set with respect to the element size at the highest pyramid level for both pairs: twice the size for the first and equal size for the second. Additionally, the regularization lengths for the gray level correction $\xi_{v}$ are set to twice the regularization length for the displacement field $\xi_{u}$ in all cases. This is motivated by the fact that the phenomena that are expected to be captured by the gray level correction (e.g., beam hardening) are of much longer wavelength (slowly varying) than those pertaining the displacement.

Finally, no initialization is provided since the multiresolution isotropic approach is capable of handling such step by means of the lower pyramid levels.

\subsection{Metric differences}

The results from the first volume pair are used to reveal their "metric differences". This can be achieved through the analysis of the found displacement field as well as the analysis of the computed relative strains. Figures $3 \mathrm{a}$ and $3 \mathrm{~b}$ showcase some slices of the analyzed volumes, as well as the kinematic decomposition used for the analysis.

To begin with, the correctness of the correlation results must be verified with the image of residuals $\eta_{u}$, shown in figure $3 \mathrm{c}$. Here are highlighted all the details that the correlation procedure was not able to register, such as the inner yarn "texture" which corresponds to the carbon fibres (filaments) bundled together. However, with the present analysis being carried out at the meso-scale, the target is to capture the yarn displacement while disregarding the complex behavior each carbon fibre exhibits. This choice is implicitly made when selecting the kinematic decomposition. Registration can be considered successful in finding the proper displacement field that aligns both volumes. Additionally, the corrected (back-deformed) image $\tilde{g}_{u}$, shown in figure $3 \mathrm{~d}$, provides a nice tool for visualizing the DVC result.

Then, as it can be seen in figure 4, the obtained (continuous) displacement field is known in the entire ROI. Since such full-field measurement can lead to an extensive and complex analysis, a more convenient representation is sought so as to better identify the occurring phenomena. Thus a virtual FE model is built 
from the tomographic acquisition of the test sample using an in-house tool [34] that accommodates the resin and each yarn as different entities. Therefore, the projection of the computed fields onto the new model allow a detailed analysis of the yarn behavior by concealing that of the resin.

In particular, the computed relative strains projected onto the model depict two highly localized distinct behaviors. The first one, seen in figures $5 \mathrm{a}$ to $5 \mathrm{c}$, characterize respectively the relative compression and dilation that experience some regions in the test volume (so as to resemble the reference). The second phenomenon, depicted in figures $5 \mathrm{~d}$ to $5 \mathrm{f}$, corresponds to a shearing effect between yarn layers.

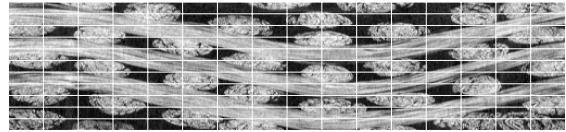

(a) $f$

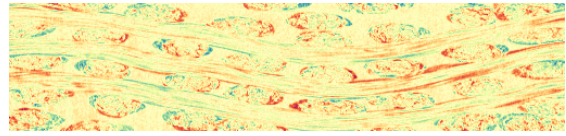

(c) $\eta_{u}$

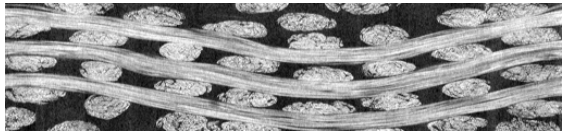

(b) $g$

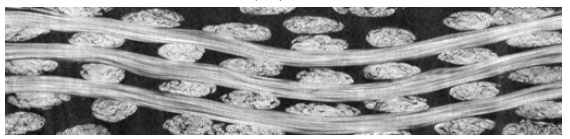

(d) $\tilde{g}_{u}$

Figure 3: Slices $(y-z)$ for the first pair of volumes analyzed for extracting the "metric differences". The slices shown for the reference image (a) and test image (b) are "equivalent", nonetheless the textile is not completely aligned. The residual image (c) validates the registration with the good alignment of yarns and only highlights the inner differences between yarns. The corrected image (d) is, as expected, properly aligned with the reference. The mesh used for kinematic decomposition is overlayed on the reference image.

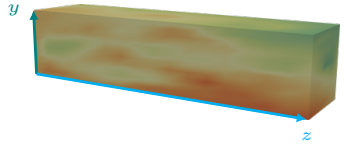

(a) $u_{x}$

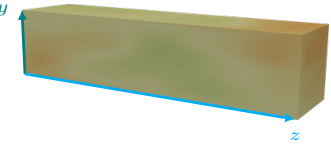

(b) $u_{y}$

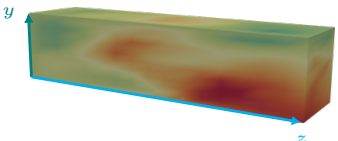

(c) $u_{z}$

Figure 4: Found displacement field $\boldsymbol{u}$ (in pixels) for the first pair of images. The $x, y$ and $z$ components of the vector field are shown in (a), (b) and (c) respectively.

\subsection{Topological differences}

The results from the second volume pair are used to reveal their "topological differences". This can be achieved through the analysis of the different images of residuals $\eta_{u}$ and $\eta_{u v}$ obtained with equations (19) and (20) respectively. 


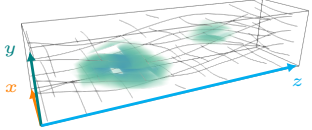

(a) $\varepsilon_{x x}$

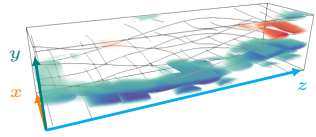

(d) $\varepsilon_{x y}$

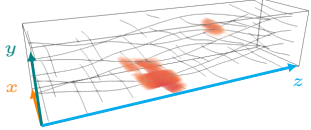

(b) $\varepsilon_{z z}$

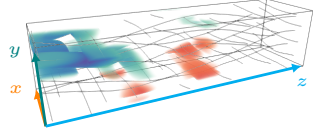

(e) $\varepsilon_{y z}$

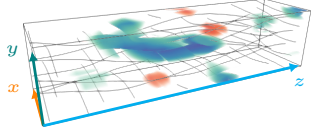

(c) $\operatorname{tr}(\varepsilon)$

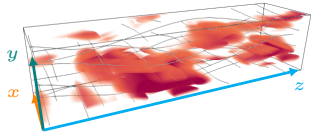

(f) $\varepsilon_{e q}$

Figure 5: Diverse relative strain fields that characterize the found metric differences between first pair of volumes by projecting them onto a known virtual model of the textile. The chosen normal relative strains highlight the volume change in the $x$ direction, in the $z$ direction and in all directions simultaneously, these are shown in (a), (b) and (c) respectively. The remaining relative strains expose the type of deformation at constant volume normal to the $z$ direction, normal to the $x$ direction and invariant to the orientation, these are shown in (d), (e) and (f) respectively.

As companion to these indicators, it can be useful to introduce the residual

$$
\eta_{o}(\boldsymbol{x})=g(s \boldsymbol{R} \boldsymbol{x}+\boldsymbol{t})-f(\boldsymbol{x})
$$

which merely accounts for a simple rigid registration thanks to the (isotropic) scaling $s$, the rotation matrix $\boldsymbol{R}$ and the translation vector $\boldsymbol{t}$.

It should be noted that the rigid adjustment observed between samples can be traced back to factors extrinsic to the manufacturing process. These include the physical placement of the samples inside the tomograph, the manual intervention usually carried out during a reconstruction process, or even the final manipulation of the reconstructed volumes. Generally, these are estimated prior to any correlation procedure using a cross-correlation measure. Here they were instead inferred from the found displacement field $\boldsymbol{u}$ using a Procrustes analysis, i.e. a posteriori. As such, it was found that only a small translation in one dimension was required for aligning this volume pair (rotation and scaling were negligible). Here is included a convenient indicator for the analysis of "metric differences" in addition to the relative strain tensor and its invariants introduced earlier.

At this moment, in view of the flagrant anomaly introduced by the missing yarns, the "simple" analysis of the residual $\eta_{o}$ seems a sensible proposition. This can be observed with the slices $(y-z)$ shown in figures $6 \mathrm{a}$ and $6 \mathrm{~b}$ as well as in the (orthogonal) slices $(x-z)$ in figures $7 \mathrm{a}$ and $7 \mathrm{~b}$. However, as it can be seen in the corresponding slice for the initial residual $\eta_{o}$ in figure $6 \mathrm{e}$, the multiple spurious high valued regions forbids such reasoning. All these zones are altogether unrelated to the topology and thus hide the actual zones of interest.

Conversely, the residual $\eta_{u}$ leads to a radically different analysis. As it can be seen in the slices $(y-z)$ and $(x-z)$ shown in figures $6 \mathrm{f}$ and $7 \mathrm{~d}$ respectively, only 
the structures that contravene the assumption of topology are accentuated. This fact is even clearer on the volumetric representation shown in figure 8a. These "topological differences" are not only highly located but also highly structured (into the shape of yarns). Furthermore, they easily distinguish between "additional" yarns with respect to the reference image (high positive residual values) and the ones missing (high negative residual values). As well as offering a clear differentiation between high valued residuals which hold a clear meaning and the multiple spurious low valued regions which do not. This represents an effortless separation between the unanticipated phenomena and the anticipated ones, such as gaps not produced by missing yarns but rather by the textile definition.

Furthermore, the residual $\eta_{u v}$ verifies that the algorithm correctly identifies the proper correction fields $\boldsymbol{u}(\boldsymbol{x}), v_{0}(\boldsymbol{x})$ and $v_{1}(\boldsymbol{x})$. After all, the minimization of this residual is precisely what is being sought, and thus is expected to tend to zero. In fact, the residual $\eta_{u v}$ is centred at zero with a standard deviation of $4.5 \%$ of the initial 16 bits dynamic range. Alike the previous volume pair, the inner yarn "texture" as well as the yarn boundaries contribute with non-zero residual values, as the slices $(y-z)$ and $(x-z)$ show in figures $6 \mathrm{~g}$ and $7 f$. Moreover, the remaining high-valued residuals are easily traced back to two sources: a ring artefact and some high density particles. These can be identified on the volumetric representations shown in figures $8 \mathrm{~b}$ and $8 \mathrm{c}$ respectively. This confirms that the isotropic multiresolution approach is robust enough even in the presence of considerable reconstruction artefacts. Finally, as it can be inferred from the slices $(y-z)$ shown in figure 9 , the fields $v_{0}(\boldsymbol{x})$ and $v_{1}(\boldsymbol{x})$ work together so as to remove the clear cupping artefact and to handle the "topological differences".

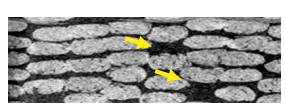

(a) $f$

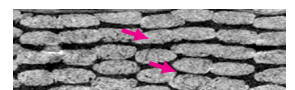

(b) $g$

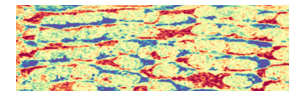

(e) $\eta_{o}$

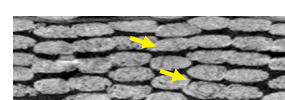

(c) $\tilde{g}_{u}$

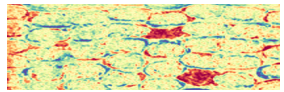

(f) $\eta_{u}$

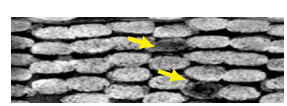

(d) $\tilde{g}_{u v}$

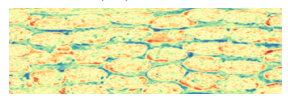

(g) $\eta_{u v}$

Figure 6: Slices $(y-z)$ for the second pair of volumes analyzed for extracting the "topological differences". The slices are chosen so as to showcase the reference image (a) and the test image (b) at "equivalent" positions. The corrected images are obtained considering the kinematic only (c) and considering all the corrections (d). The residual images are obtained considering rigid body motion only (e), considering the kinematic only (f) and considering all the corrections $(\mathrm{g})$. The arrows point to the location of two extracted yarns and their topologically equivalent ones. The "topological differences" can be observed as the yarn cross-sections highlighted in the residuals due to kinematic only. 


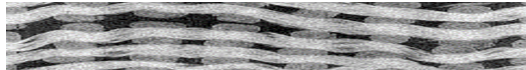

(a) $f$

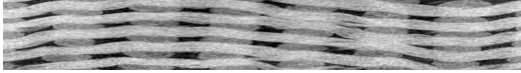

(c) $\tilde{g}_{u}$

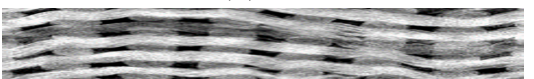

(e) $\tilde{g}_{u v}$

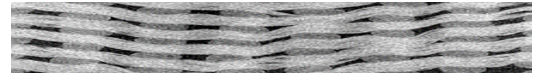

(b) $g$

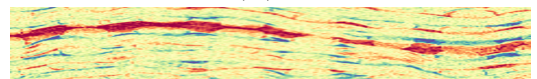

(d) $\eta_{u}$

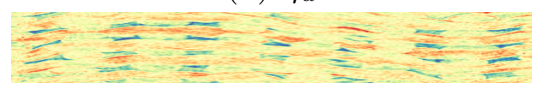

(f) $\eta_{u v}$

Figure 7: Slices $(x-y)$ for the second pair of volumes analyzed for extracting the "topological differences". Here is provided an alternative (orthogonal) view of those shown in figure 6 . The "topological difference" can be observed as the yarn highlighted in the residuals due to kinematic only.

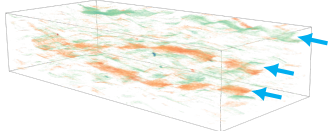

(a) $\left|\eta_{u}\right| \geq 15 \%$

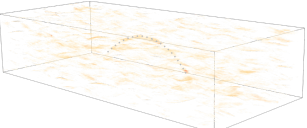

(b) $\left|\eta_{u v}\right| \geq 8 \%$

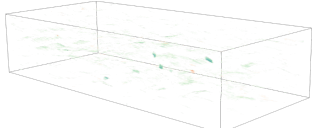

(c) $\left|\eta_{u v}\right| \geq 15 \%$

Figure 8: Volumetric representations of the residual image at different threshold values with respect to the initial dynamic range. These are obtained considering the kinematic only (a) and considering all the corrections with either a low threshold (b) or a higher one (c). The "topological differences" (pointed at by the arrows) can be observed as the solid three-dimensional structures highlighted in the residuals due to kinematic only.

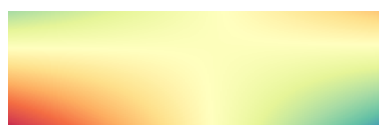

(a) $a(\boldsymbol{x})$

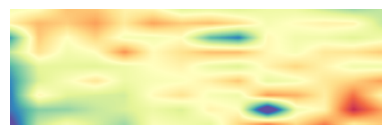

(b) $b(\boldsymbol{x})$

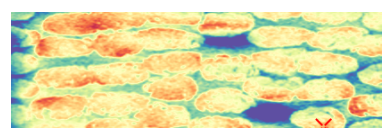

(c) $\tilde{g}_{u v}-\tilde{g}_{u}$

Figure 9: Slices $(y-z)$ for the correction fields involved in the analysis for extracting the "topological differences". The first (a) and second (b) order contributions amount to a complete (c) contribution to the solution. The latter corresponds to removing the "additional" yarns by subtracting them from the test image, as well as accounting for the cupping artefact.

\section{Conclusions and outlook}

Digital Volume Correlation under the multiresolution isotropic approach is employed for a comparative analysis of 3D woven composites. This method allows the measurement of "metric differences" and "topological differences". The former quantify yarn deformations in the studied sample, while the latter handle 
weaving anomalies, such as loops and missing yarns. These can subsequently be re-purposed for the extraction of quantitative indicators such as the relative strains, as well as qualitative ones such as the residual images. Furthermore, the proposed formulation that results into these "differences" allows analyzing each type of phenomenon independently, without interference from the other (e.g., variation of contrast due to beam hardening will not hinder the analysis relative strains), provided that the regularization has been well tuned.

This full-field analysis takes advantage of the abundant information provided in the simplest manner: using a reference. This a priori embeds multiple (complex) observations without the need to explicitly (manually) address them. Not only does it inform on current the state of the sample, but rather on its entire history. This provides a better insight into the variability of the manufacturing processes.

It should be noted that the choice of a reference (which may not be "ideal") could be considered as a fragility of the approach, since the results are only relative in nature. For this reason, the obtained results should always be subjected to a careful examination. For example, when a strong and localized strain is revealed, it is important to determine which one of the two volumes is locally satisfactory, and which one is not. Additionally, it is possible, from a long series of such analyses, to evaluate a mean displacement field. This would allow to compute a virtual reference volume (that would not exist physically). Such a choice may lead to a more solid interpretation of large differences (be they "metric" or "topological") of a studied volume as acceptable or not.

Other means of including the textile information are either of local nature $[35,36]$, which does not embed the global textile definition and thus risks betraying its topology, or they require multiple parameters $[37,38]$ so as to have a solid foundation. The approach presented here profits from the structured nature of woven composites without the need of further "custom" descriptors. Additionally, the procedure proves to be robust enough even in the presence of sizeable "topological differences" and reconstruction artefacts.

This technique has many possible applications for the analysis of composite materials. For instance, the quantitative feedback of weaving deformations can be put in relation with any of the manufacturing parameters [39] (weaving, forming, injection). This can help reducing the number of iterations needed for achieving a particular complex textile structure. Alternatively, the technique can serve as a tool for NDT in which extreme "metric differences" (that surpass some threshold) in conjunction with pertinent "topological differences" can be used for detecting and identifying weaving anomalies. In such case, a specific (manufactured) composite sample can be selected as a standard and then be used as reference for any test samples using the procedure presented here.

Finally, this method can be swiftly improved just by reformulating the reference configuration. As such, a reference sample can be replaced with a virtual textile model that corresponds to the ideal weaving pattern. This form is usually easily accessible through virtual modelling of the element (FE, CAD). Evidently, this has the advantage of removing all the phenomena related to the manufacturing process that would otherwise be interpreted by the algorithm as a priori 
corresponding to the textile. Moreover, this provides a wider perspective by consider the test sample as in a deformed or warped configuration with respect to the ideal configuration. These ideas are currently being explored and will be presented in future works.

\section{Acknowledgements}

This work is supported by the PRC MECACOMP, project co-founded by DGAC and Safran Group, involving Safran group companies, ONERA, CNRS and other academic partners. AMQ acknowledges the support of a $\mathrm{PhD}$ grant from ANRT and Safran Tech.

[1] Safran Aircraft Engines, A technological LEAP forward, URL https://www. safran-aircraft-engines.com/commercial-engines/ single-aisle-commercial-jets/leap/leap, 2016.

[2] L. Lee, S. Rudov-Clark, A. P. Mouritz, M. K. Bannister, I. Herszberg, Effect of weaving damage on the tensile properties of three-dimensional woven composites, Composite Structures 57 (1-4) (2002) 405-413, 10.1016/ S0263-8223(02)00108-3.

[3] P. Boisse, B. Zouari, A. Gasser, A mesoscopic approach for the simulation of woven fibre composite forming, Composites Science and Technology 65 (34) (2005) 429-436, ISSN 02663538, 10.1016/j.compscitech.2004.09.024.

[4] H. A. Bruck, S. R. McNeill, M. A. Sutton, W. H. Peters, Digital image correlation using Newton-Raphson method of partial differential correction, Experimental mechanics 29 (3) (1989) 261-267.

[5] B. K. Bay, T. S. Smith, D. P. Fyhrie, M. Saad, Digital volume correlation: Three-dimensional strain mapping using X-ray tomography, Experimental Mechanics 39 (3) (1999) 217-226, ISSN 0014-4851, 10.1007/BF02323555.

[6] B. K. Bay, Methods and applications of digital volume correlation, The Journal of Strain Analysis for Engineering Design 43 (8) (2008) 745-760, ISSN 0309-3247, 10.1243/03093247JSA436.

[7] M. A. Sutton, J. H. Yan, V. Tiwari, H. W. Schreier, J. J. Orteu, The effect of out-of-plane motion on 2D and 3D digital image correlation measurements, Optics and Lasers in Engineering 46 (10) (2008) 746-757, 10.1016/j.optlaseng.2008.05.005.

[8] F. Hild, S. Roux, Digital image correlation from displacement measurement to identification of elastic properties - a Review, Strain 42 (2) (2006) 69-80, ISSN 00392103, 10.1111/j.1475-1305.2006.00258.x.

[9] R. Gras, H. Leclerc, F. Hild, S. Roux, J. Schneider, Identification of a set of macroscopic elastic parameters in a $3 \mathrm{D}$ woven composite: Uncertainty analysis and regularization, International Journal of Solids and Structures 55 (2015) 2-16, ISSN 00207683, 10.1016/j.ijsolstr.2013.12.023. 
[10] G. Besnard, F. Hild, S. Roux, Finite-element displacement fields analysis from digital images: Application to Portevin-Le Châtelier bands, Experimental Mechanics 46 (6) (2006) 789-803, ISSN 00144851, 10.1007/ s11340-006-9824-8.

[11] Michel Grediac, F. Hild, Digital Image Correlation, in: M. Grédiac, F. Hild (Eds.), Full-Field Measurements and Identification in Solid Mechanics, chap. 6, John Wiley \& Sons, ISBN 978-1-118-57847-6, 496, 2012.

[12] S. Roux, F. Hild, P. Viot, D. Bernard, Three-dimensional image correlation from X-ray computed tomography of solid foam, Composites Part A: Applied Science and Manufacturing 39 (8) (2008) 1253-1265, ISSN 1359835X, 10.1016/j.compositesa.2007.11.011.

[13] H. Leclerc, J. N. Périé, S. Roux, F. Hild, Voxel-Scale Digital Volume Correlation, Experimental Mechanics 51 (4) (2011) 479-490, ISSN 00144851, 10.1007/s11340-010-9407-6.

[14] F. Hild, S. Roux, Comparison of local and global approaches to digital image correlation, Experimental Mechanics 52 (9) (2012) 1503-1519.

[15] E. Süli, D. F. Mayers, An introduction to numerical analysis, Cambridge university press, 2003.

[16] A. N. Tikhonov, V. Y. Arsenin, Solutions of ill-posed problems, Winston, 1977.

[17] T. Taillandier-Thomas, S. Roux, T. F. Morgeneyer, F. Hild, Localized strain field measurement on laminography data with mechanical regularization, Nuclear Instruments and Methods in Physics Research, Section B: Beam Interactions with Materials and Atoms 324 (2014) 70-79, ISSN 0168583X, 10.1016/j.nimb.2013.09.033.

[18] T. Taillandier-Thomas, S. Roux, F. Hild, A soft route toward 4D tomography, Physical Review Letters 117 (2) (2016) 25501, 10.1103/PhysRevLett. 117.025501 .

[19] D. Claire, F. Hild, S. Roux, A finite element formulation to identify damage fields: the equilibrium gap method, International Journal for Numerical Methods in Engineering 61 (2) (2004) 189-208.

[20] S. Roux, F. Hild, H. Leclerc, Mechanical assistance to DIC, Procedia IUTAM 4 (2012) 159-168, ISSN 22109838, 10.1016/j.piutam.2012.05.018.

[21] J. Réthoré, S. Roux, F. Hild, An extended and integrated digital image correlation technique applied to the analysis of fractured samples, European Journal of Computational Mechanics 18 (3-4) (2009) 285-306, ISSN 17797179, 10.3166/ejcm.18.285-306. 
[22] B. Wattrisse, A. Chrysochoos, J. M. Muracciole, M. Nemoz-Gaillard, Analysis of strain localization during tensile tests by digital image correlation, Experimental Mechanics 41 (1) (2001) 29-39, ISSN 0014-4851, Doi10.1007/Bf02323101.

[23] F. Hild, B. Raka, M. Baudequin, S. Roux, F. Cantelaube, Multiscale displacement field measurements of compressed mineral-wool samples by digital image correlation, Applied optics 41 (32) (2002) 6815-6828.

[24] S. Choi, S. P. Shah, Measurement of deformations on concrete subjected to compression using image correlation, Experimental Mechanics 37 (3) (1997) 307-313.

[25] M. Bornert, F. Brémand, P. Doumalin, J. C. Dupré, M. Fazzini, M. Grédiac, F. Hild, S. Mistou, J. Molimard, J. J. Orteu, L. Robert, Y. Surrel, P. Vacher, B. Wattrisse, Assessment of digital image correlation measurement errors: Methodology and results, Experimental Mechanics 49 (3) (2009) 353-370, ISSN 00144851, 10.1007/s11340-008-9204-7.

[26] P. J. Burt, E. H. Adelson, The Laplacian Pyramid as a Compact Image Code, IEEE Transactions on Communications 31 (4) (1983) 532-540, ISSN 00906778, 10.1109/TCOM.1983.1095851.

[27] B. M. H. Romeny, Front-end vision and multi-scale image analysis: multiscale computer vision theory and applications, written in mathematica, vol. 27, Springer Science \& Business Media, 2008.

[28] M. Ansar, W. Xinwei, Z. Chouwei, Modeling strategies of 3D woven composites: A review, Composite Structures 93 (8) (2011) 1947-1963, ISSN 02638223, 10.1016/j.compstruct.2011.03.010.

[29] H. Hencky, Ober die Form des ElastizitUtsgesetzes bei ideal elastischen Stoffen, Journal of Rheology 2 (1928) 169-176.

[30] S. Onaka, Appropriateness of the Hencky Equivalent Strain as the Quantity to Represent the Degree of Severe Plastic Deformation, MATERIALS TRANSACTIONS 53 (8) (2012) 1547-1548, 10.2320/matertrans. M2012077.

[31] S. C. Shrivastava, J. J. Jonas, G. Canova, Equivalent strain in large deformation torsion testing Theoretical and practical considerations, J. Mech. Phys. Solids 30 (2) (1982) 75-90.

[32] S. Shrivastava, C. Ghosh, J. J. Jonas, A comparison of the von Mises and Hencky equivalent strains for use in simple shear experiments, Philosophical Magazine 92 (18) (2012) 2264-2271, ISSN 14786435, 10.1080/14786435. 2012.671551. 
[33] M. Barburski, I. Straumit, X. Zhang, M. Wevers, S. V. Lomov, Micro-CT analysis of internal structure of sheared textile composite reinforcement, Composites Part A: Applied Science and Manufacturing 73 (2015) 45-54, ISSN 1359835X, 10.1016/j.compositesa.2015.03.008.

[34] G. Hello, J. Schneider, Z. Aboura, Numerical Simulations of Woven Composite Materials With Voxel-FE Models, in: 16th European Conference on Composite Materials (ECCM 2014), June, ISBN 9780000000002, 22-26, 2014.

[35] N. Naouar, E. Vidal-Sallé, J. Schneider, E. Maire, P. Boisse, Meso-scale $\mathrm{FE}$ analyses of textile composite reinforcement deformation based on Xray computed tomography, Composite Structures 116 (1) (2014) 165-176, ISSN 02638223, 10.1016/j.compstruct.2014.04.026.

[36] N. Naouar, E. Vidal-Salle, J. Schneider, E. Maire, P. Boisse, 3D composite reinforcement meso F.E. analyses based on X-ray computed tomography, Composite Structures 132 (2015) 1094-1104, 10.1016/j.compstruct.2015. 07.005 .

[37] S. V. Lomov, A. V. Gusakov, G. Huysmans, A. Prodromou, I. Verpoest, Textile geometry preprocessor for meso-mechanical models of woven composites, Composites Science and Technology 60 (11) (2000) 2083-2095, ISSN 02663538, 10.1016/S0266-3538(00)00121-4.

[38] I. Verpoest, S. V. Lomov, Virtual textile composites software WiseTex: Integration with micro-mechanical, permeability and structural analysis, Composites Science and Technology 65 (15-16 SPEC. ISS.) (2005) 25632574, ISSN 02663538, 10.1016/j.compscitech.2005.05.031.

[39] L. M. Dangora, C. J. Mitchell, J. A. Sherwood, Predictive model for the detection of out-of-plane defects formed during textile-composite manufacture, Composites Part A: Applied Science and Manufacturing 78 (2015) 102-112, ISSN 1359835X, 10.1016/j.compositesa.2015.07.011. 